\title{
The relation of amino-terminal propeptide of type III procollagen and severity of coronary artery disease in patients without myocardial infarction or hibernation
}

\author{
Yen-Hung Lin ${ }^{\text {a,b }}$, Yi-Lwun Ho ${ }^{\text {b,f,1 }}$, Tzung-Dau Wang ${ }^{\text {b }}$, Chung-Pin Liu ${ }^{\text {b,h }}$, Hsien-Li Kao ${ }^{\text {, }}$, \\ Chia-Lun Chao ${ }^{\text {, }}$, Kuo-Liong Chien ${ }^{\text {b }}$, Chi-Sheng Hung ${ }^{\text {, }}$, Vin-Cent Wu ${ }^{\text {a }}$, I-Jung Tsai ${ }^{\text {, }}$ \\ Ruoh-Fang Yen ${ }^{\mathrm{e}}$, Yu-Chien Shiau ${ }^{\mathrm{g}}$, Wen-Jone Chen ${ }^{\mathrm{b}, \mathrm{c}, *, 1}$ \\ ${ }^{a}$ Department of Internal Medicine, National Taiwan University Hospital Yun-Lin Branch, Yun-Lin, Taiwan \\ ${ }^{\mathrm{b}}$ Division of Cardiology, Department of Internal Medicine, \\ National Taiwan University Hospital and National Taiwan University College of Medicine, Taipei, Taiwan \\ ${ }^{c}$ Department of Emergency Medicine, National Taiwan University Hospital and National Taiwan University College of Medicine, Taipei, Taiwan \\ ${ }^{\mathrm{d}}$ Department of Pediatrics, National Taiwan University Hospital and National Taiwan University College of Medicine, Taipei, Taiwan \\ ${ }^{\mathrm{e}}$ Department of Nuclear Medicine, National Taiwan University Hospital and National Taiwan University College of Medicine, Taipei, Taiwan \\ ${ }^{\mathrm{f}}$ Department of Internal Medicine, Far-Eastern Memorial Hospital, Taipei, Taiwan \\ ${ }^{g}$ Department of Nuclear Medicine, Far-Eastern Memorial Hospital, Taipei, Taiwan \\ ${ }^{\mathrm{h}}$ Department of Internal Medicine, Yuan's General Hospital, Kaoshung, Taiwan
}

Received 29 October 2005; received in revised form 30 April 2006; accepted 15 May 2006

Available online 13 July 2006

\begin{abstract}
Objectives: The association of cardiac fibrosis and coronary artery disease (CAD) in patients without infarction or hibernation is unclear. We investigated the relationship between serum concentrations of procollagen propeptides and severity of CAD in such patients.

Design and methods: Forty-six patients (32 men; mean age 64 years) with chest pain were enrolled. All patients received stress thallium-201 single photon emission computed tomography (SPECT) and analysis of the serum levels of the amino-terminal propeptide of type I and III procollagen (PINP and PIIINP).

Results: In patients with thallium-201 perfusion defects, the number of diseased vessels was associated significantly with PIIINP $(p=0.024)$ rather than PINP $(p=0.613)$. Follow-up serum PINP and PIIINP levels after coronary intervention (mean 84 days) revealed no significant decrease.

Conclusions: Serum PIIINP level is significantly associated with the severity of CAD in patients without myocardial infarction or hibernation. (C) 2006 The Canadian Society of Clinical Chemists. All rights reserved.
\end{abstract}

Keywords: Coronary artery disease; PINP; PIIINP; Myocardial fibrosis

\section{Introduction}

Cardiac myocytes are surrounded by an extracelluar matrix consisting mainly of type I and III collagen [1]. The serum markers of collagen I and III are amino-terminal propeptides of

\footnotetext{
* Corresponding author. Division of Cardiology, Department of Internal Medicine, National Taiwan University Hospital and National Taiwan University College of Medicine, No. 7, Chung-Shan South Road, Taipei 10016, Taiwan. Tel.: +886223562168.

E-mail address: jone@ha.mc.ntu.edu.tw (W.-J. Chen).

${ }^{1}$ These authors contributed equally to this work.
}

type I and III procollagen (PINP and PIIINP). They are both valuable markers of myocardium collagen synthesis and turnover [2-4]. Excess myocardial collagen deposition has been related to several pathological conditions, including cardiac hypertrophy, myocardial hibernation, myocardial infarction (MI) and congestive heart failure [5-10]. However, there have been no data about fibrosis of ischemic myocardium in patients without MI or hibernation. We hypothesized that severity of coronary artery disease (CAD) was correlated with excess myocardial collagen deposition in such patients. Therefore, we conducted the present study to (1) evaluate the association of 
cardiac fibrosis and severity of CAD in patients without prior MI or hibernation; (2) to evaluate the changes of serum markers of cardiac fibrosis in such patients after coronary intervention.

\section{Patients and methods}

\section{Study population}

We prospectively studied 46 consecutive patients aged 50 to 75 years (32 men) who were referred for chest pain evaluation. MI was excluded by history and electrocardiograms. Regional wall motion abnormalities and impaired left ventricular contractility (left ventricular ejection fraction $<50 \%$ ) were excluded by routine echocardiography. All patients underwent stress thallium-201 single photon emission computed tomography (SPECT) for evaluation of myocardial ischemia. If perfusion defect was present, coronary angiogram was performed. Patients were divided in 3 groups according to results of stress thallium-201 SPECT: patients had no thallium201 reversible perfusion defect (group 1); patients had thallium201 reversible perfusion defects but no significant stenosis in coronary angiogram (group 2); patients had both thallium-201 reversible perfusion defects and significant stenosis in coronary angiogram (group 3). Conditions associated with elevated serum concentrations of PIIINP or PINP including chronic liver disease, pulmonary fibrosis, rheumatoid arthritis, extensive wounds and metabolic bone disease were excluded after complete medical examination [11]. Additional exclusion criteria were fixed perfusion defects in stress thallium-201 SPECT, significant valvular heart disease (more than grade 2 in valvular regurgitation, any grade of valvular stenosis), coronary intervention within the previous 3 months, leukocytosis and elevated serum levels of $\mathrm{C}$-reactive protein. Blood pressure measurements were performed by trained technicians or nurses with a mercury sphygmomanometer, and the first and fifth Korotkoff sounds were recorded to represent the systolic and diastolic pressures. Three measurements were obtained on each occasion, at 5-minute intervals, and averaged. Hypertension was said to be present if the systolic blood pressure is $\geq 140$ or diastolic blood pressure $\geq 90 \mathrm{~mm} \mathrm{Hg}$ or there is use of medication for hypertension (not including diabetes patients with systolic blood pressure $<140$ and diastolic blood pressure $<90 \mathrm{~mm} \mathrm{Hg}$ receiving only angiotensin-converting enzyme inhibitor or angiotensin II receptor blocker for diabetic proteinuria). Hyperlipidemia was defined as a total cholesterol level $\geq 240 \mathrm{mg} / \mathrm{dL}$ or total triglycerides $\geq 200 \mathrm{mg} / \mathrm{dL}$ or use of medication for hyperlipidemia. Diabetes mellitus was defined as a blood glucose level of $126 \mathrm{mg} / \mathrm{dL}$ or higher after an overnight fast or use of medication for diabetes mellitus. The study was approved by the ethical committee of the National Taiwan University Hospital, and all subjects gave informed consent.

\section{Blood sampling and determination of PINP and PIIINP}

Blood sampling was obtained from peripheral vein or percutaneous artery sheath before catheterization. Serum samples to determine PINP and PIIINP were stored at $-40^{\circ} \mathrm{C}$ until assay. Serum PINP was determined by a rapid equilibrium radioimmunoassay using commercial antisera specifically directed against the amino-terminal propeptide (Orion Diagnostica, Espoo, Finland) [12]. The intra- and interassay coefficient of variations were $<7 \%$. The sensitivity of this method was $2 \mu \mathrm{g} / \mathrm{L}$. The reference range of serum PINP was $20-76 \mu \mathrm{g} / \mathrm{L}$ in men and $19-84 \mu \mathrm{g} / \mathrm{L}$ for women. Serum PIIINP was determined by a coated-tube radioimmunoassay as described using commercial antisera specifically directed against the amino-terminal propeptide (Orion Diagnostica, Espoo, Finland) [13]. The intra- and interassay coefficient of variations of serum PIIINP were $<5 \%$. The sensitivity of PIIINP was $0.3 \mu \mathrm{g} / \mathrm{L}$. The reference range of serum PINP was $2.3-6.4 \mu \mathrm{g} / \mathrm{L}$.

\section{Echocardiography}

We performed echocardiography with a commercially available machine (SONOS 5500, Philips, Andover, Massachusetts) equipped with a 3-MHz phased-array probe. Echocardiographic studies including two-dimensional M-mode and Doppler ultrasound recordings were performed. Left ventricular dimension, septum and posterior wall thickness and left ventricular ejection fraction (M-mode) were measured via parasternal long axis view according to the procedures of the American Society of Echocardiography [14]. Left ventricle was subdivided in 16 segments for detection of regional wall motion abnormalities [15]. Echocardiographic left ventricular mass index (LVMI) was calculated according to the method of Devereux et al. [16].

\section{Stress thallium-201 SPECT}

The stress thallium-201 SPECT was performed with treadmill exercise test (modified Bruce protocol) or dipyridamole injection $(0.56 \mathrm{mg} / \mathrm{kg})$. Two to $3 \mathrm{mCi}$ of thallium-201 was injected intravenously after peak exercise or $3 \mathrm{~min}$ after dipyridamole infusion was completed. Thallium SPECT was carried out within $10 \mathrm{~min}$ (stress phase) and at $4 \mathrm{~h}$ (redistribution phase) after the injection of thallium-201 using a large field gamma camera (Starcam 3000, General Electric, Milwaukee, Wisconsin) equipped with a low-energy, allpurpose collimator. The detector collected data through a $180^{\circ}$ arc from the $45^{\circ}$ right anterior oblique to the $45^{\circ}$ left posterior oblique position. Contiguous transaxial tomograms were reconstructed into 6-mm-thick multiple sections, using a filtered back-projection method with a Hanning ramp filter. Thereafter, the tomographic slices were reorganized to obtain standard short-axis, horizontal long-axis and vertical long-axis views for analysis.

\section{SPECT analysis}

All SPECT images were interpreted qualitatively by 2 experienced observers. We used the 16-color scale in image interpretation. The left ventricle was divided into 10 segments, as proposed by Iskandian et al. [17] Thallium- 
201 activity in each segment was graded as 3 (normal), 2 (mild reduced), 1 (moderately reduced) or 0 (severely reduced or absent). A segment was considered abnormal if its grade was 2 or less [18]. Stress images were compared with redistribution images to evaluate the presence or absence of reversible or fixed perfusion defects. Reversible perfusion defect was defined as abnormal segments with increment of tracer uptake $>1$ grade at the redistribution phase compared with the stress phase. Fixed perfusion defect was defined as absence of tracer uptake at stress and redistribution phase. The kappa value for inter-observer agreement for SPECT interpretation was 0.90 [19].

\section{Cardiac catheterization and reperfusion therapy}

Diagnostic coronary angiogram was performed in patients with reversible perfusion defect in stress thallium-201 SPECT. A computer-aided quantitative angiographic analysis system (DCI-S Automated Coronary Analysis System, Philips Medical System, Eindhoven, Netherlands) was used. A $\geqq 50 \%$ diameter stenosis was considered significant. The indications of percutaneous coronary intervention (PCI) were (1) diameter stenosis $\geqq 70 \%$ or (2) diameter stenosis $\geqq 50 \%$ with perfusion defect in the responding coronary territory in stress thallium201 SPECT. Procedural heparin was given at $70 \mathrm{U} / \mathrm{kg}$, and the activated coagulation time was kept at 250 to $300 \mathrm{~s}$. The goal of PCI was to achieve a final residual diameter stenosis $\leqq 20 \%$, and stenting was allowed and depended on operators. In patient with multiple vessel disease, staging PCI was allowed for safety reasons. All patients received antiplatelet therapy. Aspirin (100 $\mathrm{mg}$ /days) was started at least 3 days prior to angioplasty. Clopidogrel (300 mg loading dose then $75 \mathrm{mg} /$ days) was given for at least 6 weeks in patients receiving stents. In patients with 3 vessel or left main disease, the choice of PCI or coronary artery bypass graft surgery (CABG) was depended on operators and patients.

\section{Follow-up study}

All patients were followed monthly at the outpatient clinic. At 12 weeks, blood sampling to determine PINP and PIIINP was obtained again from patients receiving reperfusion therapy. If restenosis was suspected clinically, follow-up angiography was mandated to confirm the diagnosis.

\section{Statistical analysis}

Data were expressed as median (range). Comparisons between groups for continuous data were made using the Mann-Whitney $U$ test or Kruskal-Wallis 1-way ANOVA. Differences between proportions were assessed by chi-square test or Fisher's Exact Test. Univariate general linear model was used to determine relationship between diseased vessel number and PINP or PIIINP levels. Multivariate linear regression test with stepwise subset selection was performed to find out various factors associated with PIIINP levels. The factors for analysis included age, sex, hypertension, diabetes mellitus, hyperlipidemia, smoking, left ventricle ejection fraction, left ventricle mass index and diseased vessel numbers. The entry and removal thresholds for stepwise selection were 0.1 and 0.2 , respectively. Wilcoxon signed ranks test was performed to compare the prePCI and post-PCI levels of PINP or PIIINP. A value of $p<0.05$ was considered to indicate statistical significance.

\section{Results}

\section{Clinical data, echocardiographic results and serum concentrations of PINP and PIIINP}

The clinical data, echocardiographic results and serum concentrations of PINP and PIIINP are summarized in Table 1. The age of the whole population, men and woman were 63.5 $(50-75)$ years, $64(50-75)$ years and 62.5 (54-74) years,

Table 1

Patient characteristics

\begin{tabular}{|c|c|c|c|c|}
\hline & Group $1(n=9)$ & Group $2(n=13)$ & Group $3(n=24)$ & $P$ value \\
\hline PINP ( $\mu \mathrm{g} / \mathrm{L})$ & $25.38(16.59-61.98)$ & $33.69(23.70-90.50)$ & $39.34(6.07-74.49)$ & 0.125 \\
\hline PIIINP $(\mu \mathrm{g} / \mathrm{L})$ & $3.60(2.89-6.85)$ & $3.84(1.49-4.89)$ & $4.31(1.9-12.26)$ & 0.362 \\
\hline Age (years) & $64(50-75)$ & $59(54-75)$ & $64.5(51-74)$ & 0.220 \\
\hline LVEF (\%) & $68(63-76)$ & $68(54-75)$ & $65.5(51-74)$ & 0.297 \\
\hline LVMI $\left(\mathrm{g} / \mathrm{m}^{2}\right)$ & $127.40(89.79-190.54)$ & $117.19(68.46-174.02)$ & $120.78(79.94-216.87)$ & 0.224 \\
\hline Men & $6(67 \%)$ & $5(38 \%)^{\mathrm{a}}$ & $21(88 \%)$ & 0.008 \\
\hline Hyperlipidemia & $1(11 \%)^{b}$ & $4(31 \%)^{a}$ & $20(83 \%)$ & $<0.001$ \\
\hline Hypertension & $3(33 \%)$ & $7(54 \%)$ & $18(75 \%)$ & 0.075 \\
\hline Diabetes mellitus & $1(11 \%)$ & $2(15 \%)$ & $8(33 \%)$ & 0.271 \\
\hline Smoking & $4(44 \%)$ & $2(15 \%)$ & $9(38 \%)$ & 0.246 \\
\hline \multicolumn{5}{|l|}{ Medication } \\
\hline ACEI/ARB & $1(11 \%)$ & $4(31 \%)$ & $13(54 \%)$ & 0.064 \\
\hline$\beta$-blockers & $1(11 \%)^{\mathrm{c}}$ & $7(54 \%)$ & $16(67 \%)$ & 0.019 \\
\hline Statin & $1(11 \%)$ & $3(23 \%)$ & $12(50 \%)$ & 0.069 \\
\hline
\end{tabular}

$\mathrm{PINP}=$ amino-terminal propeptide of type I procollagen; PIIINP=amino-terminal propeptide of type III procollagen; LVEF=left ventricular ejection fraction; $\mathrm{LVMI}=$ left ventricular mass index; $\mathrm{ACEI}=$ angiotensin-converting enzyme inhibitor; $\mathrm{ARB}=$ angiotensin II receptor blocker.

${ }^{\mathrm{a}} p<0.01$ between group 2 and group 3 .

${ }^{\mathrm{b}} p<0.001$ between group 1 and group 3 .

${ }^{c} p<0.01$ between group 1 and group 3 . 


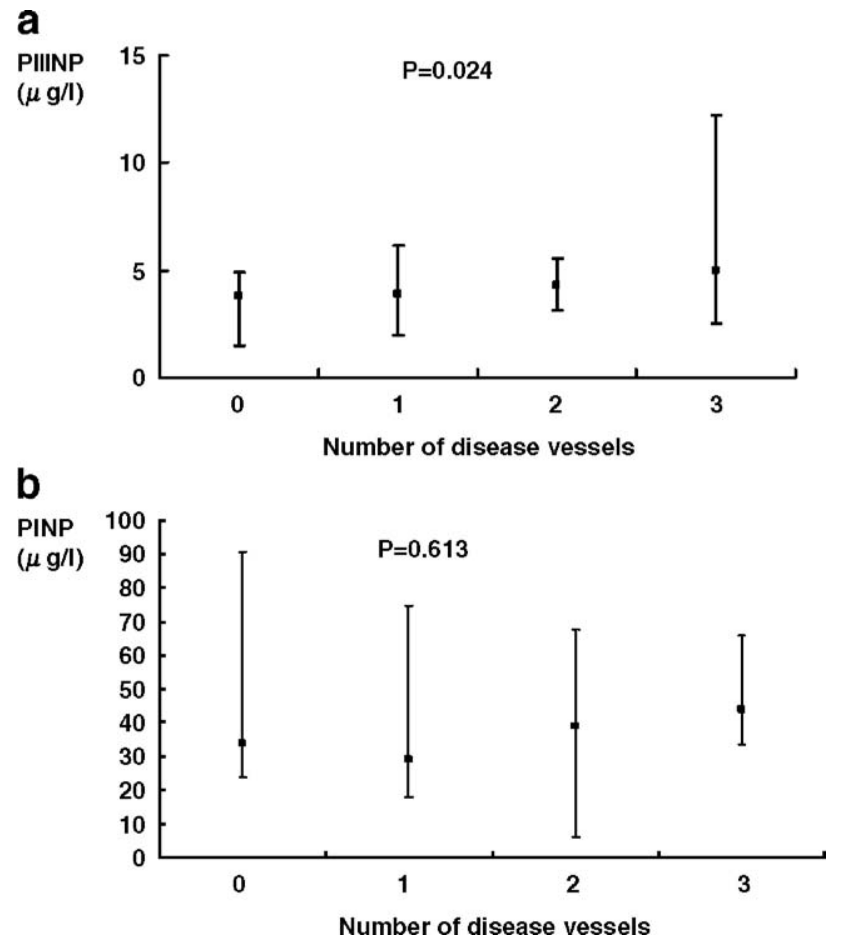

Fig. 1. (a) The relationship between diseased vessel number and amino-terminal propeptide of type III procollagen (PIIINP) level. Solid square represented the median value of PIIINP in each subgroup, and pseudobars represented the upper and lower limits of PIIINP in each subgroup. The serum levels of PIIINP in patients with 0-, 1-, 2- and 3-vessel disease were 3.84 (1.49-4.89), 3.87 (1.996.15), 4.33 (3.15-5.55) and $4.76(1.49-12.26) \mu \mathrm{g} / \mathrm{L}$, respectively. $p$ value was calculated by univariate general linear model to determine relationship between diseased vessel number and PIIINP. The intergroup comparison was performed by Mann-Whitney $U$ test and $p$ values were listed below: 0 -vessel vs. 1-vessel ( $p=0.574) ; 0$-vessel vs. 2 -vessel $(p=0.127) ; 0$-vessel vs. 3 -vessel $(p=0.127) ; 1$ vessel vs. 2 -vessel $(p=0.616) ; 1$-vessel vs. 3 -vessel $(p=0.494)$; 2 -vessel vs. 3 vessel $(p=0.699)$. (b) The relationship between diseased vessel number and amino-terminal propeptide of type I procollagen (PINP) level. Solid square represented the median value of PINP in each subgroup, and pseudobars represented the upper and lower limits of PINP in each subgroup. The serum levels of PINP in patients with 0-, 1-, 2- and 3-vessel disease were 33.69 (23.7090.50), 28.92 (18.02-74.49), 38.99 (6.07-67.73) and 43.86 (33.49-65.61) $\mu \mathrm{g} / \mathrm{L}$, respectively. $p$ value was calculated by univariate general linear model to determine relationship between diseased vessel number and PINP. The intergroup comparison was performed by Mann-Whitney $U$ test, and $p$ values were listed below: 0 -vessel vs. 1 -vessel $(p=0.406) ; 0$-vessel vs. 2 -vessel ( $p=0.639) ; 0$-vessel vs. 3 -vessel $(p=0.210) ; 1$-vessel vs. 2 -vessel $(p=0.820) ; 1$ vessel vs. 3 -vessel $(p=0.820)$; 2 -vessel vs. 3 -vessel $(p=0.699)$.

respectively. Stress thallium-201 SPECT showed no perfusion defect in 9 patients (group 1). In the other 37 patients with perfusion defects in stress thallium-201 SPECT, 13 patients had patent coronary artery in coronary angiogram (group 2), and 24 patients had significant lesion in one or more coronary arteries (group 3). No significant differences were found in age, hypertension, diabetes, smoking, left ventricular ejection fraction, left ventricular mass index, angiotensin-converting enzyme inhibitor or angiotensin II receptor blocker usage, statin usage and serum PINP and PIIINP values among three groups. There are more men in group 3 than group 2. Group 3 patients had higher percentage to have hyperlipidemia than group 1 and group 2. Group 3 patients had higher percentage to use beta blocker than group 1.
Table 2

Multivariate linear regression model of factors associated with PIIINP level (model adjust $R^{2}=0.113$ )

\begin{tabular}{|c|c|c|}
\hline & $\beta$ coefficient & $P$ valu \\
\hline Diseased vessel number $(n)$ & 0.371 & 0.024 \\
\hline
\end{tabular}

\section{Cardiac catheterization and reperfusion therapy}

In group 3 patients, 12 patients had one-vessel disease, 6 patients had 2-vessel disease and 6 patients had 3-vessel disease. Two patients with 3-vessel disease were suggested to receive $\mathrm{CABG}$, but they chose medical therapy only. PCI was performed in 28 vessels of the other 22 patients smoothly. The successful rate was $100 \%$, and the interventional goal was reached in all treated vessels. No complications or elevated level of cardiac enzyme were noted after procedure. All patients discharge smoothly the next day after PCI.

\section{Patients follow-up}

All patients underwent the scheduled follow-up without symptoms. One patient with 3 -vessel disease receiving initial medical therapy underwent $\mathrm{CABG} 6$ weeks later. In 22 patients receiving PCI, two patients refused second blood sampling. We examination the blood level of PINP and PIIINP in other 20 patients (with 33 diseased vessels and 25 vessels received PCI; follow-up time: 82 (33-149)).

\section{The association of serum level of PINP and PIIINP and CAD}

In order to find the relationship between CAD and serum level of PINP and PIIINP, linear regression analysis was performed in patients receiving coronary angiogram (group 2 and group 3, $n=37$ ). The result was shown in Figs. 1a and b. Serum PIIINP level is significantly associated with the number of diseased vessels $(p=0.024)$; the values were $3.84(1.49$ 4.89), 3.87 (1.99-6.15), $4.33(3.15-5.55)$ and 4.76 (1.4912.26) $\mu \mathrm{g} / \mathrm{L}$ in patients with $0-, 1-, 2-$ and 3 -vessel disease. There is no association with serum PINP level and number of diseased vessel $(p=0.613)$; the values were 33.69 (23.7090.50), 28.92 (18.02-74.49), 38.99 (6.07-67.73) and 43.86 (33.49-65.61) $\mu \mathrm{g} / \mathrm{L}$ in patients with $0-, 1-, 2$ - and 3-vessel disease. Multivariate linear regression test showed only the number of diseased vessels as a significant predicator of PIIINP levels (Table 2). After reperfusion therapy, serum PINP and PIIINP levels showed no significant change (Table 3).

Table 3

Serum PINP and PIIINP levels pre- and post-PCI

\begin{tabular}{lccc}
\hline & Pre-PCI & Post-PCI & $P$ value \\
\hline PINP $(\mu \mathrm{g} / \mathrm{L})$ & $38.99(6.07-74.49)$ & $31.96(20.41-81.29)$ & 0.370 \\
PIIINP $(\mu \mathrm{g} / \mathrm{L})$ & $4.04(1.99-12.26)$ & $4.29(1.70-7.37)$ & 0.204 \\
\hline
\end{tabular}

$\mathrm{PINP}=$ amino-terminal propeptide of type I procollagen; $\mathrm{PIINP}=$ amino-terminal propeptide of type III procollagen; $\mathrm{PCI}=$ percutaneous coronary intervention. 
Table 4

Serum PINP and PIIINP levels in hypertensive and normotensive patients

\begin{tabular}{llll}
\hline & $\begin{array}{l}\text { Hypertensive patients } \\
(n=28)\end{array}$ & $\begin{array}{l}\text { Normotensive patients } \\
(n=18)\end{array}$ & $P$ value \\
\hline PINP $(\mu \mathrm{g} / \mathrm{L})$ & $38.5(18.02-90.50)$ & $26.10(6.07-67.73)$ & 0.032 \\
PIIINP $(\mu \mathrm{g} / \mathrm{L})$ & $3.94(1.99-12.26)$ & $3.87(1.49-6.15)$ & 0.380 \\
\hline
\end{tabular}

$\mathrm{PINP}=$ amino-terminal propeptide of type I procollagen; $\mathrm{PIIINP}=$ amino-terminal propeptide of type III procollagen.

The association of serum level of PINP and PIIINP and hypertension

As shown in Table 4, hypertensive patients had higher serum PINP level than normotensive patients $(p=0.032)$. The serum PIIINP levels were comparable with hypertensive and normotensive patients $(p=0.380)$. In hypertensive patients, the use of ACEI/ARB also did not significantly influence the serum PINP and PIIINP levels (Table 5).

\section{Discussion}

Serum levels of procollagen propeptides are considered as useful and reliable markers of cardiac collagen turnover. They reflect the condition and activity of cardiac tissue repair and fibrosis $[2,4,8,20]$. However, the serum PINP level showed no association with severity of CAD in the present study. In our previous study, there is also no difference of serum carboxyterminal peptide of type I procollagen (PICP) level in patients with or without reversible perfusion defect in stress thallium201 SPECT [21]. Both collagen type I and III were present in cardiac tissue. Although type I collagen is predominant in myocardium, type III collagen is more specific to cardiac tissue $[22,23]$. In the study of Alla et al., serum PIIINP rather than collagen type I including PINP and PICP changed in patients with congestive heart failure [23]. In the study of Lombardi et al., patients with hypertrophic cardiomyopathy had a higher serum PIIINP, but not PINP level than control [3]. Therefore, collagen III makers, such as PIIINP, may be more specifically recommended to monitor cardiac collagen turnover.

According to Bonnet et al., patient with CAD has higher serum PIIINP than control [24]. But 17 out of 34 patients in the CAD group have previous $\mathrm{MI}$ in that study. Besides, there is absent of correlation between the severity of atherosclerosis and serum PIIINP level [24]. In the present study, we demonstrated elevated level of PIIINP in patients who had CAD but no previous MI or hibernation. The relationship of CAD and PIIINP is independent on left ventricular mass and left ventricular function. The level of PIIINP was correlated directly with severity of CAD. This phenomenon reflected that excess type III collagen synthesis was ongoing if the threshold of ischemic burden was achieved. The mechanism responsible for myocardial fibrosis in patients without MI is less understood. In an animal MI model without significant hemodynamic change, non-ischemic myocardium distant from the infarct site shows molecular evidence of myocardial fibrosis, such as upregulation of mRNAs of type III collagen [9]. Interestingly, transforming growth factor (TGF)- $\beta 1$ is also up-regulated in non-infarct zones. It shows that local myocardial ischemic injury can independently cause myocardial fibrosis associated with TGF- $\beta 1$.

There were no changes of serum levels of procollagen peptides before and after reperfusion therapy. It suggested that fibrotic activity persisted in myocardium despite reperfusion therapy. Our data were compatible with a previous study [20]. According to Host et al., 12 patients received coronary artery bypass due to symptomatic ischemic heart disease without heart failure [20]. The serum PIIINP levels increase significantly even up to 12 months post-operation.

In a recent study, Wasywich et al. did not detect an elevation and transcardiac step-up of serum PIIINP level in aortic stenosis patients and patients with stable CAD who have normal left ventricular ejection fractions [25]. It was hard to compare this report with our study due to different study population and ischemic burden. The study of Wasywich et al. was designed to detect the transcardiac step-up of serum PIIINP level in patients with stable CAD. However, no comparison was made between disease and disease-free groups The severity of CAD and the ischemic burden was also not demonstrated in that study. In our study, the myocardial ischemic was proven by stress thallium201 SPECT, and the elevation of serum PIIINP was evident in more severe CAD. Therefore, the lack of serum PIIINP increase in Wasywich's study may be due to lack of ischemic burden or less severe CAD.

In the present study, hypertensive patients had higher serum PINP than normotensive patients. Arterial hypertension stimulates both myocardial procollagen gene expression and collagen synthesis [26]. The excess deposit of myocardial collagen content results in myocardial stiffness and diastolic dysfunction $[27,28]$. The association of serum marker of type I collagen and hypertension is well known in previous reports $[2,11]$. In our previous study, hypertension patients also had higher serum PICP level than control [21]. In the present study, there was no difference of serum PIIINP values between these 2 groups. This phenomenon implied that type III collagen was not proportional to type I collagen in the myocardial collagen deposition. The finding might be explained by the much more synthesis of type I collagen than that of type III collagen during chronic pressure overload [29].

Our study has limitations. First, we did not perform endomyocardial biopsy to document fibrotic process. However, it is not ethical to perform endomyocardial biopsy in CAD patients routinely. Besides, serum markers of

Table 5

Serum PINP and PIIINP levels in hypertensive patients use or not use ACEI/ ARB

\begin{tabular}{lcll}
\hline & $\begin{array}{l}\text { Hypertensive patients use } \\
\text { ACEI/ARB }(n=15)\end{array}$ & $\begin{array}{l}\text { Hypertensive patients not } \\
\text { use ACEI/ARB }(n=13)\end{array}$ & $p$ \\
\hline PINP $(\mu \mathrm{g} / \mathrm{L})$ & $41.31(22.38-74.49)$ & $33.67(18.02-90.50)$ & 0.467 \\
PIIINP $(\mu \mathrm{g} / \mathrm{L})$ & $4.48(1.99-12.26)$ & $3.83(2.53-6.85)$ & 0.217 \\
\hline
\end{tabular}

$\mathrm{PINP}=$ amino-terminal propeptide of type I procollagen; PIIINP = amino-terminal propeptide of type III procollagen; ACEI=angiotensin-converting enzyme inhibitor; $\mathrm{ARB}=$ angiotensin II receptor blocker. 
procollagen peptides are reliable in remote monitoring collagen turnover [2-4]. Second, our study was limited by small patient numbers and relatively short follow-up period. This may yield to alpha or beta type errors in statistical analysis. Further large-scaled studies are needed. Third, we did not follow coronary angiography or stress thallium-201 SPECT routinely after revascularization procedure to exclude silent ischemia due to restenosis or newly developed lesions. These lesions may influence the serum levels of procollagen peptides after reperfusion therapy. However, the restenosis rate is low in 12 weeks, so the effect of coronary restenosis was deduced to play a minor role in our findings. Fourth, some patients were partially revascularized due to staging PCI, and this may decrease the difference of PINP or PIIINP levels before and after the revascularization procedure.

In conclusion, serum PIIINP level is significantly associated with the severity of CAD in patients without myocardial infarction or hibernation. Short-term follow-up fails to document the changes of serum PIIINP levels after reperfusion therapy.

\section{Acknowledgment}

This study was supported by National Taiwan University Hospital Yun-Lin Branch (grants NTUHYL 94.S012).

\section{References}

[1] Heeneman S, Cleutjens JP, Faber BC, et al. The dynamic extracellular matrix: intervention strategies during heart failure and atherosclerosis. J Pathol 2003;200:516-25.

[2] Lopez B, Gonzalez A, Varo N, Laviades C, Querejeta R, Diez J. Biochemical assessment of myocardial fibrosis in hypertensive heart disease. Hypertension 2001;38:1222-6.

[3] Lombardi R, Betocchi S, Losi A, et al. Myocardial collagen turnover in hypertrophic cardiomyopathy. Circulation 2003;108:1455-60.

[4] Uusimaa P, Risteli J, Niemela M, et al. Collagen scar formation after acute myocardial infarction relationship to infarct size, ventricular function, and coronary artery patency. Circulation 1997;96:2565-72.

[5] Cicoira M, Rossi A, Bonapace S, et al. Independent and additional prognostic value of amino-terminal propeptide of type III procollagen circulating levels in patients with chronic heart failure. J Card Fail 2004;10:403-11.

[6] Zannad F, Alla F, Dousset B, Perez A, Pitt B. Limitation of excessive extracellular matrix turnover may contribute to survival benefit of spirolactone therapy in patients with congestive heart failure. Circulation 2000;102:2700-6.

[7] Sutton MGSJ, Sharpe N. Left ventricular remodeling after myocardial infarction pathophysiology and therapy. Circulation 2000;101:2981-8.

[8] Poulsen SH, Host NB, Jensen SE. Relationship between serum aminoterminal propeptide of type III procollagen and change of left ventricular function after acute myocardial infarction. Circulation 2000;102:1527-32.

[9] Tsuda T, Gao E, Evangelisti L, Markova D, Ma X, Chu M. Post-ischemic myocardial fibrosis occurs independent of hemodynamic changes. Cardiovasc Res 2003;59:926-33.
[10] Elsasser A, Vogt AM, Nef H, et al. Human hibernating myocardium is jeopardized by apoptotic and autophagic cell death. J Am Coll Cardiol 2004;43:2191-9.

[11] Diez J, Laviades C, Mayor G, Gil MJ, Monreal I. Increased serum concentrations of procollagen peptides in essential hypertension. Relation to cardiac alterations. Circulation 1995;91:1450-6.

[12] Melkko J, Kauppila S, Niemi S, Risteli L, Haukipuro K, Jukkola A, et al. Immunoassay for intact amino-terminal propeptide of human type I procollagen. Clin Chem 1996;42:947-54.

[13] Risteli J, Niemi S, Trivedi P, Ma"entausta O, Mowat AP, Risteli L. Rapid equilibrium radioimmunoassay for the amino-terminal propeptide of human type III procollagen. Clin Chem 1988;34:715-8.

[14] Sahn DJ, DeMaria A, Kisslo J, Weyman A. Recommendation regarding quantitation in M-mode echocardiography: results of survey of echocardiographic measurements. Circulation 1987;58:1072-83.

[15] Schiller NB, Shah PM, Crawford M, et al. Recommendations for quantitation of the left ventricle by two-dimensional echocardiography. American Society of Echocardiography Committee on Standards, Subcommittee on Quantitation of Two-dimensional Echocardiograms. J Am Soc Echocardiogr 1989;2:358-67.

[16] Devereux RB, Reichek N. Echocardiographic determination of left ventricular mass in man: anatomical validation of the method. Circulation 1977;55:613-8.

[17] Iskandian AS, Heo J, Askenase A, Segal BL, Helfant RH. Thallium imaging with single photon emission computed tomography. Am Heart J 1987; 114:852-65.

[18] Huang PJ, Yen RF, Chieng PU, Chen ML, Su CT. Do beta-blockers affect the diagnostic sensitivity of dobutamine stress thallium-201 single photon emission computed tomographic imaging? J Nucl Cardiol 1998;5:34-9.

[19] Ho YL, Chen CL, Hsu RB, et al. Assessment of the myocardial changes in heart transplant recipients without evident acute myocardial rejection by integrated backscatter: comparison with simultaneous dobutamine stress echocardiography and Thallium-201 SPECT. Ultrasound Med Biol 2001;27:171-9.

[20] Host NB, Aldershvile J, Horslev-Petersen K, Hoyer S, Haunso S. Serum aminoterminal propeptide of type III procollagen after cardiac transplantation and the effect of rejection. Am J Cardiol 1996;78:1406-10.

[21] Lin YH, Shiau YC, Yen RF, et al. The relation between myocardial cyclic variation of integrated backscatter and serum concentrations of procollagen propeptides in hypertensive patients. Ultrasound Med Biol 2004;30:885-91.

[22] Bishop JE, Laurent GJ. Collagen turn-over and its regulation in the normal and hypertrophing heart. Eur Heart J 1995;16:38-44.

[23] Alla F, Kearney-Schwartz A, Radauceanu A, Dores SD, Dousset B, Zannad F. Early changes in serum markers of cardiac extra-cellular matrix turnover in patients with uncomplicated hypertension and type II diabetes. Eur J Heart Fail 2006;8:147-53.

[24] Bonnet J, Garderes PE, Aumailley M, et al. Serum type III procollagen peptide levels in coronary artery disease (a marker of atherosclerosis). Eur J Clin Invest 1988;18:18-21.

[25] Wasywich CA, Webster MWI, Richards AM, Stewart RAH. Coronary sinus and ascending aortic levels of aldosterone, angiotensin II, and B-type natriuretic peptide in patients with aortic stenosis and in patients with Coronary heart disease. Am J Cardiol 2006;97:1068-72.

[26] Bishop JE, Lindahl G. Regulation of cardiovascular collagen synthesis by mechanical load. Cardiovasc Res 1999;42:27-44.

[27] Diez J, Lopez B, Gonzalez A, Querejeta R. Clinical aspects of hypertensive myocardial fibrosis. Curr Opin Cardiol 2001;16:328-35.

[28] Lopez B, Gonzalez A, Varo N, Laviades C, Querejeta R, Diez J. Biochemical assessment of myocardial fibrosis in hypertensive heart disease. Hypertension 2001;38:1222-6. 